\title{
Data Base Management
}

National Cancer Institute

\section{Source}

National Cancer Institute. Data Base Management. NCI Thesaurus. Code C18729.

Database management involves the design and maintenance of electronic data storage systems to facilitate and optimize the categorization, selection, and retrieval of information. 